Rabaska

Revue d'ethnologie de l'Amérique française

Marchand, Suzanne. Partir pour la famille. Fécondité, grossesse et accouchement au Québec (1900-1950). Québec, Septentrion, 2012, 269 p. ISBN 978-2-89448-691-7

\title{
Johanne Daigle
}

Volume 11, 2013

URI : https://id.erudit.org/iderudit/1018547ar

DOI : https://doi.org/10.7202/1018547ar

Aller au sommaire du numéro

Éditeur(s)

Société québécoise d'ethnologie

ISSN

1703-7433 (imprimé)

1916-7350 (numérique)

Découvrir la revue

Citer ce compte rendu

Daigle, J. (2013). Compte rendu de [MARCHAND, SUZANnE. Partir pour la famille. Fécondité, grossesse et accouchement au Québec (1900-1950). Québec, Septentrion, 2012, 269 p. ISBN 978-2-89448-691-7]. Rabaska, 11, 240-243.

https://doi.org/10.7202/1018547ar d'utilisation que vous pouvez consulter en ligne.

https://apropos.erudit.org/fr/usagers/politique-dutilisation/ 
Marchand, Suzanne. Partir pour la famille. Fécondité, grossesse et accouchement au Québec (1900-1950). Québec, Septentrion, 2012, 269 p. ISBN 978-2-89448-691-7.

La fécondité, la grossesse et l'accouchement, ces processus biologiques que l'on a cru pendant longtemps atemporels, comptent assurément parmi les plus importants faits de société. Essentiels, ils sont codifiés et ordonnés dans pratiquement toutes les cultures. Rappelons la norme voulant qu'au Québec, l'enfant dit naturel - auparavant qualifié de bâtard pour être né alors que ses parents n'étaient pas mariés - n'ait pas eu les mêmes droits que l'enfant légitime jusqu'à la fin du $\mathrm{xx}^{\mathrm{e}}$ siècle. Les politiques familiales impliquant les autorités politiques, juridiques, médicales et religieuses encadrent la naissance. Plusieurs études ont mis l'accent sur ces politiques et prescriptions, alors que l'on connaissait bien peu l'expérience humaine de la naissance.

Dans Partir pour la famille, l'ethnologue Suzanne Marchand aborde précisément le vécu entourant la naissance au Québec. Elle replace cette expérience au cœur de la vie personnelle de gens « du peuple » ayant eu des enfants au cours de la première moitié $\mathrm{du} \mathrm{Xx}^{\mathrm{e}}$ siècle. L'expression populaire « partir pour la famille» illustre bien le changement que représente la naissance d'un enfant. Il s'agit d'un parcours, voire d'un voyage conduisant de la fécondation à l'accouchement, et de ses imparables implications. L'auteur n'a pas ménagé ses efforts pour faire parler la «majorité silencieuse » de ces questions à la fois personnelles et politiques pour « rendre compte de leurs vies, de leurs joies et de leurs peines » (p. 11). Sans négliger les études statistiques, l'accent va aux témoignages, nombreux, de femmes surtout, mais aussi de quelques hommes qui en ont parlé, jetant un faisceau de lumière sur le sens même de ce parcours dans la société québécoise.

La grande force de l'ouvrage, qui consiste à laisser parler quantité de témoins à propos de ces expériences dans leur vie, tient à l'ampleur et à la nouveauté de la recherche. Le noyau central provient de «l'extraordinaire documentation » (p. 10), comme l'écrit l'auteur, des Archives de folklore et d'ethnologie de l'Université Laval qui regroupent les enquêtes orales recueillies depuis une soixantaine d'années sur divers aspects du sujet et ce, auprès d'une cinquantaine de personnes différentes. À cet imposant corpus s'ajoutent des résultats d'enquêtes publiés par d'autres auteurs (anthropologues, sociologues, historiens et historiennes surtout) sur la grossesse, le mariage, l'adoption, l'enfance, la mort, la maladie, la médecine traditionnelle, la sexualité et j'en passe. Les témoignages proviennent en outre de récits autobiographiques et de documents personnels, mais aussi des observations faites lors d'enquêtes de terrain réalisées souvent dans de petites communautés villageoises de la province. Ce qui offre un éventail d'expériences tant 
pour les régions rurales et même isolées que pour les centres urbains dont Montréal, qui reste davantage étudié. Se greffent encore à cette documentation les brochures et manuels qui ont connu une grande diffusion auprès des parents quant aux soins et à l'éducation des enfants, ainsi que des ouvrages majeurs publiés en Europe et en Amérique du Nord sur divers aspects du sujet.

Cette somme de connaissances sur des thèmes comme la fécondité, la contraception, la stérilité, la grossesse, l'accouchement, le nouveau-né, l'enfance, la mort... nous éclaire sur les attentes, les espoirs et les inquiétudes liées à la parentalité, en particulier à la maternité, sur les croyances aussi, lesquelles permettent d'expliquer l'origine de certaines pratiques dont quelques-unes subsistent aujourd'hui. Par exemple, l'idée qu'il faille éviter les émotions fortes pendant la grossesse. Au cours de la première moitié du $\mathrm{xx}^{\mathrm{e}}$ siècle, on craignait que l'enfant à naître ne porte une marque sur le corps ou une déformation témoignant de l'émotion ressentie par sa mère. Les neuf chapitres que compte l'ouvrage traitent de trois étapes du processus étudié, y compris des facteurs qui en empêchent la marche : la fécondité, la contraception et l'avortement, et la stérilité ; la grossesse, les attentes et espoirs qu'elle suscite et l'expérience de l'accouchement ; la naissance pour l'enfant et les croyances et pratiques concernant le « petit enfant». Le dernier chapitre est consacré à la mortalité infantile et maternelle, des drames encore trop fréquents dans la période étudiée. Ce plan de démonstration fort judicieux permet de prendre en compte, dans une même réalité, des croyances et des pratiques, des attentes et des difficultés de l'expérience. Derrière chacune des parties de l'ouvrage, on retrouve des explications des phénomènes étudiés et des pistes de réponse à des questions de portée universelle, dont celles-ci : « Comment les futurs parents vivaient-ils l'attente de l'enfant à naître ?» ; «Rêvaient-ils d'avoir un garçon ou une fille?»; « Les femmes avaient-elles peur de mourir en donnant la vie ?» ; «L'allaitement était-il plus populaire que le biberon ? »; "Comment [la mortalité infantile] cette triste réalité était-elle vécue ?» (p. 17).

Les réponses à de telles questions, clairement expliquées, nous apprennent par exemple qu'il existait des couples stériles et ce, en proportion significative (entre $12 \%$ et $14 \%$ des femmes nées entre 1887 et 1913 n'ont pas eu d'enfant), une triste réalité alors que la fécondité était vue comme une " preuve de plénitude physique »; que la grossesse était une période de vie chargée d'inquiétude et, si les femmes consultaient peu les médecins pour cette raison, elles prenaient par contre maintes précautions en faveur de l'enfant à venir; que les parents avaient souvent honte de donner naissance à un enfant handicapé et que certains de ces enfants étaient éliminés à la naissance par un « médecin compatissant »; que les garçons jugés plus utiles à la campagne 
étaient souvent préférés aux filles et que, même dans les familles nombreuses, la mort d'un enfant ou pire, d'une mère de famille, était un événement triste, sinon dramatique.

Suzanne Marchand relève de façon remarquable le défi d'offrir une synthèse des connaissances sur cet ensemble de phénomènes reliés à la naissance, une synthèse inédite réalisée en mettant à profit une foule d'expériences diversifiées. En cherchant à donner un sens à cet ensemble disparate, l'auteur en offre une analyse fine. La pertinence des courtes introductions et des conclusions des chapitres qui tissent des liens entre les différentes sections de l'ouvrage, tout en resserrant l'explication qui unit le propos, en témoigne bien. L'écriture fluide et l'aisance dans le récit révèlent par ailleurs la grande maîtrise d'un sujet pourtant encore rempli d'embûches et d'incertitudes. Parmi les embûches, soulignons l'utilisation de témoignages puisés dans divers corpus rendant difficile, sinon impossible, de distinguer l'incidence de facteurs tels les milieux géographiques (ville-campagne par exemple) et sociaux (groupes sociaux, suivant les métiers et les situations familiales spécifiques), les années considérées (la période étudiée reste vaste), l'âge des parents et les temps concernés par leurs expériences de vie et, bien sûr, les aspects concernant spécifiquement le traitement des nombreux témoignages utilisés.

L'œuvre de synthèse, si nécessaire, a pourtant ses limites. D'autant que l'abondante production scientifique, foisonnante depuis quelques années sur divers aspects du vaste sujet traité, rendait également difficile une mise à jour de l'information. La terminologie utilisée pour rendre compte des témoignages mis à profit s'en ressent. Les termes récurrents d' " informateur» et « informatrice » et à plus forte raison d' « informatrices interrogées » (p. 112) gagneraient à être revus au profit de termes plus actuels (témoins, narrateurs, et autres) du fait que l'enquête orale de type qualitatif découle généralement d'une relation entre deux personnes dans laquelle l'une témoigne de son expérience spécifique. Il ne s'agit assurément pas d'un interrogatoire. Quant aux zones d'incertitudes, l'auteur relève les plus importantes en conclusion, à savoir le manque de connaissances du travail de celles qui officiaient comme « sages-femmes », sans formation spécifique mais expérimentées, et de la perception des hommes quant à la paternité et aux processus entourant la fécondité, la grossesse et l'accouchement. Les témoignages d'hommes étant beaucoup moins nombreux que ceux des femmes sur ces questions.

En dépit de ces quelques inconvénients, le choix de s'adresser à un vaste public en présentant un ouvrage de synthèse accessible par l'écriture fluide, les nombreux encadrés, les illustrations éloquentes et les vignettes explicatives claires et instructives, peut-être issue de l'exposition sur le sujet, est une rareté. Cela est d'autant plus vrai que l'ouvrage est remarquablement fouillé et finement analysé. On ne voit plus la thèse qui sert de fondement 
scientifique. L'ouvrage éclaire à lui seul un vaste pan de la culture populaire au Québec en abordant des questions universelles, à partir de croyances et de pratiques spécifiques, dont témoignent des personnes qui n'ont guère laissé d'autres traces de leur vécu. Cette somme d'expériences vécues dans une période de transition dans l'univers de la naissance, brillamment synthétisées et explicitées par Suzanne Marchand, nous montre, plus largement, comment s'opère le changement social. Cet ouvrage majeur, de lecture aisée, instructive et même fascinante, deviendra assurément un incontournable sur le sujet.

Johanne Daigle

Université Laval

Mathieu, François. Les Cloches d'église du Québec. Sujets de culture. Québec, Septentrion, 2010, 212 p. ISBN 978-2-89448-605-4.

L'auteur François Mathieu, bachelier en philosophie et en arts plastiques de l'Université Laval, détient aussi une maîtrise en études québécoises de l'Université du Québec à Trois-Rivières, dont le livre Les Cloches d'église du Québec. Sujets de culture en constitue le mémoire. À travers l'histoire campanaire du Québec, l'auteur met en lumière le potentiel expressif et symbolique des cloches d'église de même que leur valeur matérielle et identitaire, comme patrimoine à préserver et à mettre en valeur. La recherche des plus pertinentes menée par l'auteur vient enrichir la documentation québécoise dans le domaine plus vaste du patrimoine religieux du Québec. Le bilan fort intéressant que nous offre l'auteur est accessible à tous. Le livre se subdivise en quatre chapitres, précédés d'un avant-propos et d'une brève introduction.

La première partie de l'ouvrage esquisse un portrait historique de la campanologie tant européenne que québécoise, des fondeurs itinérants de la Nouvelle-France aux grandes entreprises de France et d'Angleterre. Ce chapitre souligne la valeur identitaire et le potentiel symbolique de la cloche. Comme le précise l'auteur, « ce qui autrefois était qualifié d'identitaire parce qu'il était religieux pourrait être vu comme identitaire en tant que culturel et historique » (p. 26). Pour lui, la cloche est un acteur important de l'histoire civile et politique d'un pays et, à ce titre, elle a un rôle culturel à jouer. Cette première partie propose donc une réflexion sur la cloche comme sujet de culture.

La deuxième partie présente la cloche dans ses aspects matériels et immatériels, à la fois témoin matériel du patrimoine, en tant qu'objet d'artisan et instrument de musique, et témoin du patrimoine immatériel à travers la pratique d'un savoir-faire. La cloche y est montrée comme un objet singulier, 